\title{
ARTICLE
}

Received 6 Aug 2012 | Accepted 30 May 2013 | Published 25 Jun $2013 \quad$ DOl: 10.1038/ncomms3074

\section{Molecular adsorption induces the transformation of rhombohedral- to Bernal-stacking order in trilayer graphene}

Wenjing Zhang ${ }^{1, \star}$, Jiaxu Yan ${ }^{2, \star}$, Chang-Hsiao Chen ${ }^{1}$, Liu Lei ${ }^{3}$, Jer-Lai Kuo ${ }^{1}$, Zexiang Shen² \& Lain-Jong $\mathrm{Li}^{1,4}$

The Bernal ( $A B A$ )-stacked graphene trilayer is presumed to be thermodynamically more stable than the rhombohedral $(A B C)$ counterpart. However, the thermal transformation from $A B C$ to $A B A$ domains does not occur at a temperature lower than $1,000^{\circ} \mathrm{C}$. Here we report that $A B C$-stacked trilayers are transformed to $A B A$-stacked layers after an organic molecule triazine is evaporated onto graphene surfaces at $150^{\circ} \mathrm{C}$. The transformation is found to always initiate at the $A B A-A B C$ domain boundaries. Simulations based on density function theory considering the van der Waals interaction suggest that after triazine decoration the energy difference between $A B A$ and $A B C$ domains is larger, providing a driving force for stacking transformation. The molecular dynamics simulation results further suggest that the triazine decoration on the wrinkles at the $A B C-A B A$ domain boundary activates the wrinkle sliding toward the $A B C$ domains, leading to the stacking transformation from $A B C$ to $A B A$.

\footnotetext{
${ }^{1}$ Institute of Atomic and Molecular Sciences, Academia Sinica, Taipei 10617, Taiwan. ${ }^{2}$ Centre for Disruptive Photonic Technologies, School of Physics and Mathematic Sciences, Nanyang Technological University, Singapore 637371, Singapore. ${ }^{3}$ State Key Laboratory of Luminescence and Applications, Changchun Institute of Optics, Fine Mechanics and Physics, Chinese Academy of Sciences, Changchun 130033, China. ${ }^{4}$ Department of Physics, National Tsing Hua University, HsinChu 300, Taiwan. ${ }^{*}$ These two authors contributed equally to this work. Correspondence and requests for materials should be addressed to L.-J.L. (email: lanceli@gate.sinica.edu.tw) or to Z.X.S. (email: zexiang@ntu.edu.sg).
} 
S ingle-layer and bilayer graphene sheets have stimulated intense interest because of their unique electronic structures $^{1-4}$ and great potential for various applications, such as high-frequency devices, ${ }^{5}$ flexible electronics ${ }^{6,7}$ and sensors ${ }^{8,9}$. Few-layer graphene sheets, including trilayer and other thicker graphene layers, have recently attracted increasing attention ${ }^{10-16}$, because the crystallographic stacking order of individual graphene sheets provides an additional parameter for tuning their electronic properties ${ }^{17-21}$. The rhombohedral $(A B C)$ and Bernal (ABA) stacking orders are two stable crystallographic configurations. These graphene trilayers are expected to exhibit different phenomena at low charge densities because of various layer-to-layer electronic interactions and competing symmetries ${ }^{15}$. The ABA-stacking order is slightly more thermodynamically stable than the ABC-stacking order. However, the $\mathrm{ABC}$ graphite was found to retain its stacking order, even at a temperature as high as $1,000-1,200^{\circ} \mathrm{C}$ (refs 22,23 ). These results indicate a significant energy barrier for configuration transformation from the ABC to ABA-stacking order. Lui et al. ${ }^{17}$ demonstrated that the Raman 2D peak shape of the ABC-stacked graphene trilayer (and tetralayer) was distinctively different from that of the ABA-stacked layer, where the full-width at half maximum (FWHM) of the ABC-stacked few-layer graphene was $\sim 10 \mathrm{~cm}^{-1}$ broader as compared with the ABA-stacked graphene. Cong et al. ${ }^{24}$ reported that the $\mathrm{M}$ band at $\sim 1,740 \mathrm{~cm}^{-1}$ is a single and asymmetric peak in the ABAstacked trilayer, but it splits into two peaks in the ABC-stacked trilayer. These studies provide a simple and useful methodology for characterizing the $\mathrm{ABC}$ - and $\mathrm{ABA}$-stacked domains by Raman spectroscopy.

In this contribution, a layer of small organic molecules 1,3 , 5-triazine (abbreviated as triazine) was deposited onto mechanically exfoliated few-layer graphene by vapour evaporation at only $150^{\circ} \mathrm{C}$. After triazine decoration, certain ABC-stacked domains were transformed to ABA-stacked domains. This was confirmed by the changes in three Raman vibration modes of $2 \mathrm{D}, \mathrm{M}$ and $\mathrm{G}$ bands. The experiments revealed that the transformation always started at the $\mathrm{ABA}-\mathrm{ABC}$ domain boundaries, where the domain conversion (growth of $\mathrm{ABA}$ from $\mathrm{ABC}$ domains) is analogous to the conventional crystal growth process.

\section{Results}

Identification of $\mathrm{ABC}$ and $\mathrm{ABA}$ domains. Figure 1a shows the optical micrograph for a graphene flake obtained by micromechanical exfoliation. Figure $1 \mathrm{~b}$ presents the atomic force microscopy (AFM) topology for the graphene flake, indicated as the selected squared area in Fig. 1a. The AFM image in Fig. 1b shows that the selected sample is a trilayer graphene with uniform thickness $(1.32 \mathrm{~nm})$ across the whole area. The Raman 2D mode, arising from an inter-valley double-resonant process that involves two in-plane transverse optical phonons near the $\mathbf{K}$ point in the Brillouin zone, is sensitive to the electronic structure of graphene ${ }^{23}$. Its line shape provides an accurate signature of the AB-stacked bilayer graphene ${ }^{25,26}$. Lui et al. ${ }^{17}$ demonstrated that the $2 \mathrm{D}$ bandwidth of the $\mathrm{ABC}$-stacked few-layer graphene was broader than that of the ABA-stacked graphene. Figure 1c displays the Raman 2D peak profiles, excited by a 473-nm laser, for the graphene flake shown in Fig. 1b. The flake also consists of two types of Raman 2D shapes, which corresponds to ABA- and ABC-stacked graphene trilayers. The 2D FWHM of the ABAstacking order is $\sim 8 \mathrm{~cm}^{-1}$ smaller than that of the ABC-stacking order. Lui et al. ${ }^{17}$ suggested fitting the $2 \mathrm{D}$ spectrum at each pixel of the spatial mapping to a single Lorenzian function. Displaying the FWHM of the fitted Lorenzian peak for each pixel visualizes the spatial distribution of the $\mathrm{ABC}$ - and ABA-stacked domains.
Figure 1d shows the Raman 2D FWHM mapping of the trilayer flake based on Lui's method. Two distinct areas are identified as the ABA- and ABC-stacked domains. These two areas are undistinguishable in the AFM height image (Fig. 1b). In addition to the 2D FWHM, the Raman M band at $\sim 1,740 \mathrm{~cm}^{-1}$, which has been assigned to the second-order overtone of the out-ofplane transverse mode ${ }^{25,26}$, is also the fingerprint of the interlayer coupling effect. The $\mathrm{M}$ band is activated by the strong coupling between the graphene layers, and thus, it is observed in the ABstacked graphene bilayer and absent in both the monolayer graphene and the incommensurate bilayer graphene ${ }^{23,25-26}$. Cong et l. $^{24}$ have recently shown that the $M$ band at $\sim 1,740 \mathrm{~cm}^{-1}$ exhibits a single peak in the ABA-stacked trilayer, but it splits into two peaks in the ABC-stacked trilayer. Figure 1e shows the Raman M-band features for the identified ABA- and ABCstacked trilayers obtained from the same flake. The $M$ band for the ABA domain has a single peak and is centred at $1,746 \mathrm{~cm}^{-1}$, whereas it splits into two peaks $\left(1,735\right.$ and $\left.1,751 \mathrm{~cm}^{-1}\right)$ in the $\mathrm{ABC}$ domain, consistent with the experimental observation by Cong et al. ${ }^{24}$ Supplementary Figure S1 provides the M-band characteristics for the bilayer, trilayer and tetralayer graphene. The integrated peak area of the split $M$ bands from the ABCstacked domain is smaller than that of the ABA-stacked domain, and the false colour spatial mapping constructed by plotting the $\mathrm{M}$ peak area also allows visualizing the spatial distribution of ABA- and ABC-stacked domains, as shown in Fig. 1f. Figure $1 \mathrm{~g}$ shows that the $\mathrm{G}$ peak frequency for the ABAstacking order is $3 \mathrm{~cm}^{-1}$ higher than that of the ABC-stacking order, consistent with the report by Lui et al. ${ }^{17}$. The ABCand $\mathrm{ABA}$-domain distribution identified by the integrated $\mathrm{M}$ peak area or G-band frequency is the same as that identified by the 2D FWHM.

Domain transformation. The ABA-stacking order is slightly more thermodynamically stable compared with the ABC-stacking order $^{27,28}$. The lateral energy barrier between these two stackings has been calculated to be $\sim 2 \mathrm{meV}$ per atom ${ }^{29}$. A recent study confirmed that the ABC-stacked graphene trilayer was stable after annealing at $800^{\circ} \mathrm{C}$ (ref. 19). Previously, we have found that an organic molecule, triazine can be adsorbed and form a uniform layer on bilayer graphene, resulting in the band-gap opening by breaking the inversion symmetry between the top and bottom layers ${ }^{30}$. With this inspiration, we thermally evaporated triazine molecules on the $\mathrm{ABC}$-stacked graphene trilayer at $150^{\circ}$ for $8 \mathrm{~h}$. Figure $1 \mathrm{i}$ shows the Raman 2D spectra of the ABA- and ABCdomains for the same flake after triazine decoration, where the FWHM of the previously identified ABC domain is obviously narrowed, and the peak shape becomes undistinguishable to the $\mathrm{ABA}$ domains. The Raman spectrum for the unconverted $\mathrm{ABC}$ domains after triazine decoration was also included for comparison from another trilayer flake on the same substrate. The unconverted $\mathrm{ABC}$ domain still exhibits a broader FWHM after triazine decoration. Figure $1 \mathrm{k}$ demonstrates that the doublepeaked $\mathrm{M}$ band in pristine $\mathrm{ABC}$ domains has been changed to a single peak after triazine decoration. Similarly, the G-band frequency for the $\mathrm{ABC}$ domain has become the same as that for the ABA domain after triazine decoration, as shown in Fig. $1 \mathrm{~m}$. The Raman mappings for 2D FWHM, the integrated M-band area and the G-band frequency shown in Fig. 1j,l,n clearly shows that the ABC-stacking domain has been changed to an ABA domain, where the false colour scale for each mapping is the same as in the corresponding Fig. 1d,f,h. This study performed the $150{ }^{\circ} \mathrm{C}$ triazine decoration experiments for seven samples, and the transformation was observed in all samples. The transformation probability is $41.3 \%$ on average. A higher decoration temperature 
a

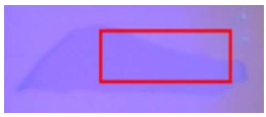

C

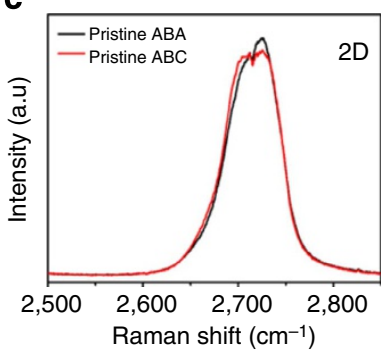

d
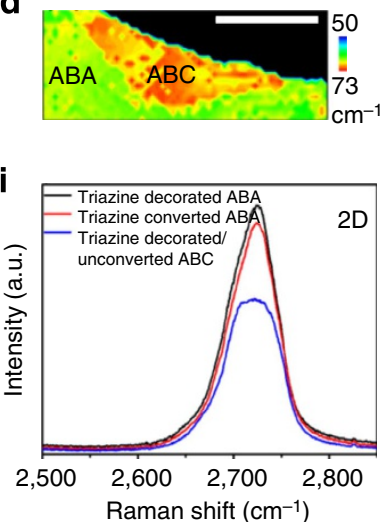

j

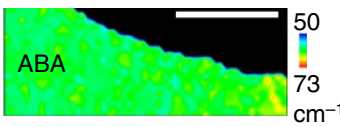

b
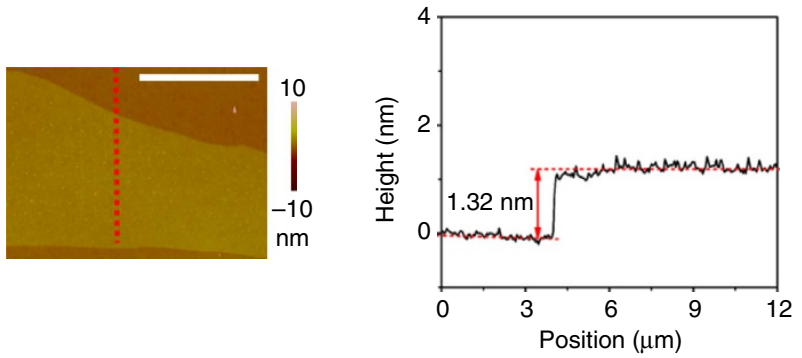

e

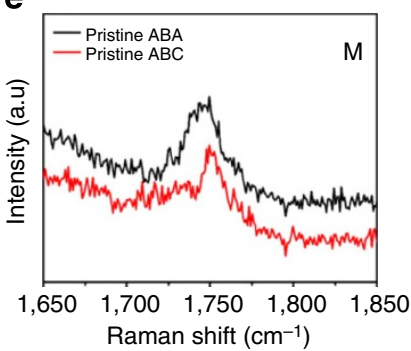

g

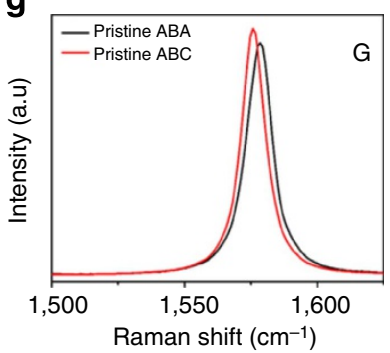

h
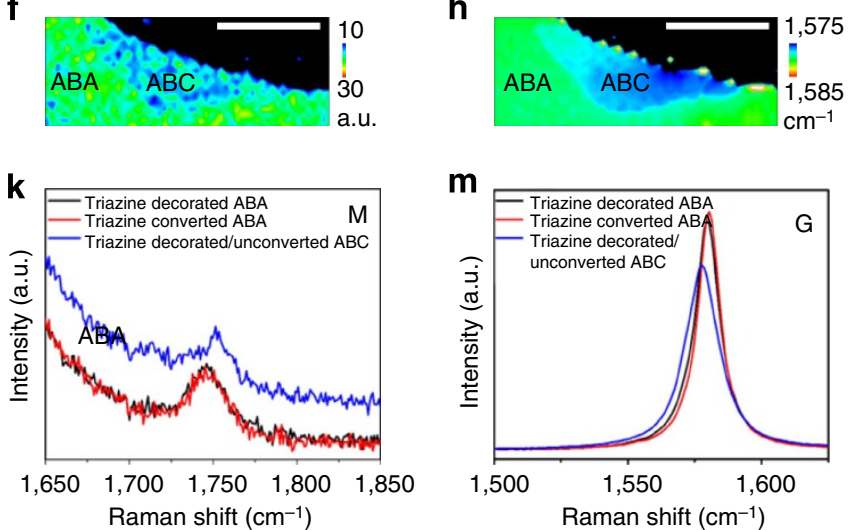

m

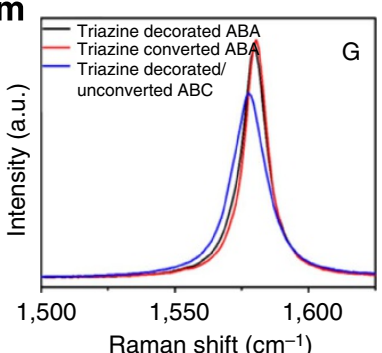

I

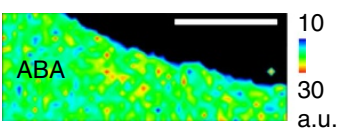

n

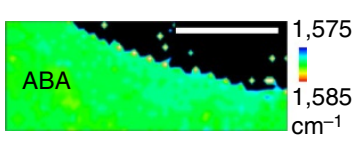

Figure 1 | Characterization of ABC and ABA domains using Raman spectroscopy. (a) The optical micrograph of a graphene trilayer with $A B A$ and $A B C$ domains. (b) The AFM height image for the around ABC-stacked area of the red square in (a). The cross-sectional height along the dotted line in (b) shows on the figure to the right that there is no height difference between $A B A$ and $A B C$ domains. (c,e,g) Raman 2D, M and G-band spectra, respectively, for the pristine $A B A$ - and $A B C$-stacking domains. (d,f,h) Raman mappings of the pristine trilayer constructed using FWHM of 2D band, integrated M-band peak area and G-band frequency, respectively. (i,k, $\mathbf{m}$ ) Raman 2D, M and G-band spectra, respectively, for the triazine decorated ABA, triazine converted $A B A$ and triazine decorated/unconverted $A B C$-stacking domains. (j,l,n) Raman mappings of the sample after triazine decoration, constructed using 2D FWHM, integrated M-band peak area and G-band frequency, respectively. The scale bar length on the right of the AFM and mappings, $10 \mu \mathrm{m}$.

of $250^{\circ} \mathrm{C}$ results in a lower $(9.6 \%)$ transformation, because the desorption of triazine molecules from the graphene surface is faster at higher temperatures (see Supplementary Table S1 for details). We have tested two other commonly used molecules, tetracyanoquinodimethane and melamine, known to exhibit strong interaction with graphene. However, no stacking transformation was observed.

The results in Fig. 1 suggest that the Raman mapping of the 2D FWHM suffices in efficiency to differentiate between the ABA and $\mathrm{ABC}$ domains. As the intensity of the $2 \mathrm{D}$ band is strong, $2 \mathrm{D}$ FWHM Raman mapping was used to further reveal the details of the stacking order transformation. Figure 2a,b shows the Raman 2D FWHM map and the AFM topology image for the selected area of another graphene flake before triazine deposition. Figure 2c,d shows the corresponding Raman map and AFM image after triazine decoration. The ABA domains remain as $\mathrm{ABA}$ after triazine deposition. However, the location labelled with a 'cross' sign originally identified as the ABC-stacking order has been transformed to ABA-stacking, directly proving that the ABA-stacking order is thermodynamically more stable than the ABC-stacking order. The newly formed ABA domain starts from the small ABA domain (the green triangle area in Fig. 2a). Supplementary Figure S2 provides another example showing that the conversion started from ABA domains. To ensure that the 2D and M-band changes after triazine deposition are not the artifact caused by triazine, the triazine layer was removed by annealing at 
a

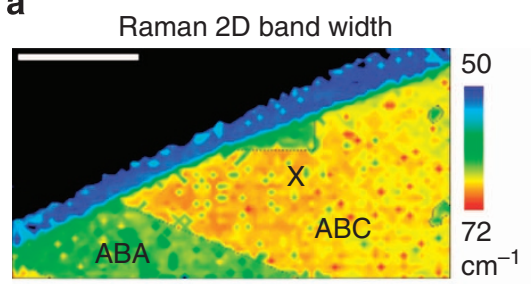

b

$\downarrow$ Triazine decoration

c

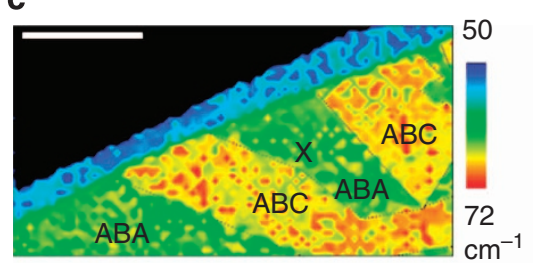

d

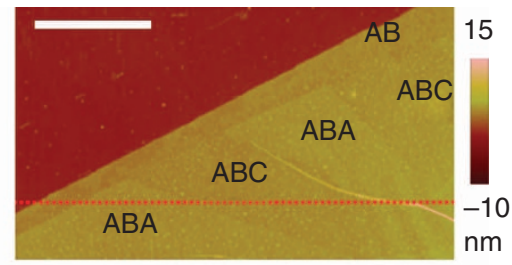

$500{ }^{\circ} \mathrm{C}$ annealing

e

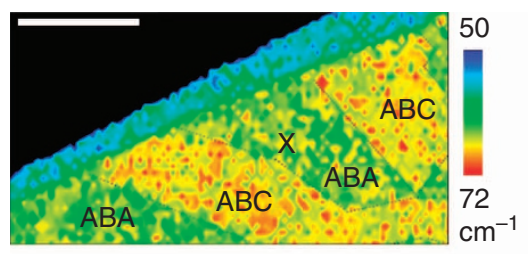

f

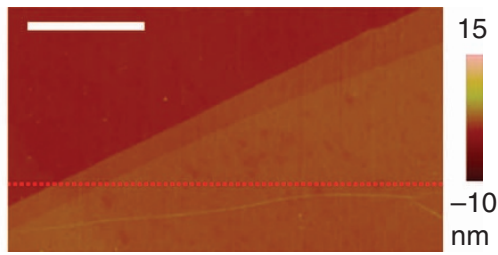

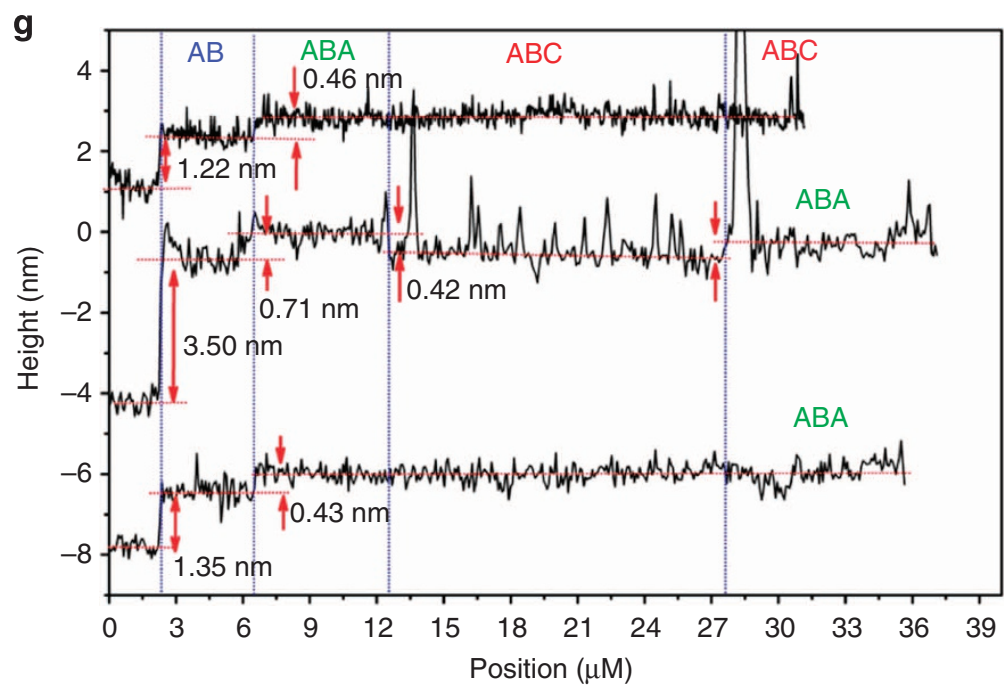

Figure 2 | Transformation of stacking order in trilayer graphene. $(\mathbf{a}, \mathbf{c}, \mathbf{e})$ Raman mappings of the FWHM of 2D band before and after triazine decoration, and after triazine removal by $500^{\circ} \mathrm{C}$ annealing, respectively. The black dotted line is to guild to visualize the boundaries between the $A B A$ and $A B C$ domains. $\mathbf{b}, \mathbf{d}, \mathbf{f}$ Are the AFM images corresponding to the Raman mapping areas in the a,c,e, respectively. (g) The AFM height profiles for a selected line across the $A B A$ and $A B C$ domains. The scale bars on the left of (a-f), $10 \mu \mathrm{m}$ in length.

$500^{\circ} \mathrm{C}$ (in Ar for $5 \mathrm{~h}$ ). Supplementary Figure S3a,b shows the $2 \mathrm{D}$ and $\mathrm{M}$ bands for the converted domain before and after triazine removal, respectively. The line shape of the $2 \mathrm{D}$ band and the $\mathrm{M}$-band profile remain unchanged, corroborating that the $\mathrm{ABC}$ stacked domain was transformed to an ABA-stacked domain. Figure 2e,f shows the corresponding Raman map and AFM image after triazine removal, where the AFM topography of the whole area becomes flat again because of the removal of triazine molecules. However, the $\mathrm{ABA}$ and $\mathrm{ABC}$ domain distribution is the same as before triazine removal.
Morphology of $\mathrm{ABA}$ and $\mathrm{ABC}$ domains. Figure $2 \mathrm{~g}$ shows the height profiles of a selected line across the ABA- and ABC-stacked areas shown in Fig. 2b,d,f. The top trace shows that the ABA- and ABC-stacked domains are of similar height and undistinguishable in the AFM topological image. After triazine decoration, the thickness of the AB bilayer area increases from 1.22 to $3.5 \mathrm{~nm}$, and the height difference between the $\mathrm{AB}$ bilayer and the ABA trilayer increases from 0.46 to $0.71 \mathrm{~nm}$. After triazine decoration, the $\mathrm{ABA}$-stacked trilayer domain is $0.42 \mathrm{~nm}$ thicker than the ABC-stacked trilayer domain. These results 
a

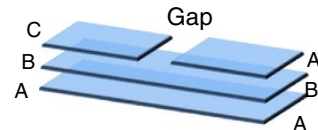

Model I

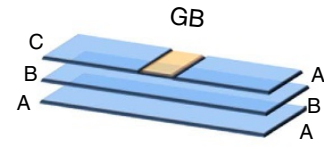

Model II

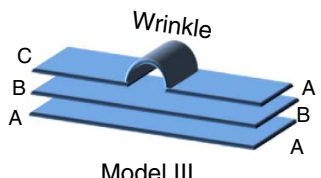

Model III

b

C
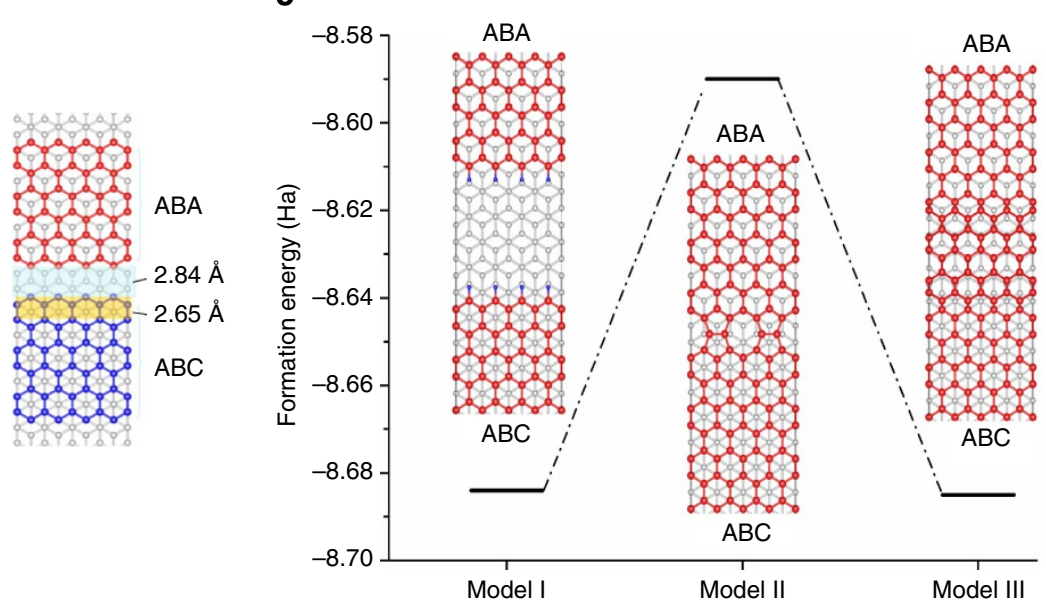

Figure 3 | Proposed models for constructing graphene trilayers. (a) Schematic illustration for three possible ways of constructing an $A B A / A B C$ graphene trilayer. Model I: $A B A / A B C$ separated by a gap. Model II: $A B A / A B C$ separated by $G B$ defects. Model III: $A B A / A B C$ separated by a wrinkle. (b) To construct a $A B A / A B C$ domain structure, the carbon atoms between $A B A$ and $A B C$ domains must shift by $2.84+2.65 \times n \AA(n=0,1,2,3, \ldots)$ along the armchair direction. (c) The formation energies $\left(E_{\mathrm{f}}\right)$ for all these three possible configurations, Model I, II and III. $E_{\mathrm{f}}=E_{\mathrm{system}}-m E_{\mathrm{C}}-n E_{\mathrm{H}}$, where $E_{\mathrm{C}}$ and $E_{\mathrm{H}}$ denote the energies of bulk graphite and hydrogen molecule.

corroborate that a triazine layer was deposited onto the graphene surfaces. The AFM topological image of the ABA- and ABCstacked domains (Fig. 2d) corresponds well to the mapping of the Raman 2D bandwidth (Fig. 2c), meaning that the AFM topological image after triazine decoration could also be used to visualize the spatial distribution of $\mathrm{ABA}$ - and $\mathrm{ABC}$-stacked domains.

\section{Discussion}

We have compared the total energies of a triazine molecule adsorbed parallel to or perpendicularly on a trilayer graphene sheet for both ABA and ABC-stacking forms, as shown in Supplementary Table S2. Our results show that a triazine molecule prefers to absorb parallel to a trilayer graphene sheet. To further clarify the effect of triazine decoration, this study performed density functional theory (DFT) simulations to examine whether the $\mathrm{ABC}$ to $\mathrm{ABA}$ domain transformation is energetically favourable. As shown in Supplementary Table S3, the local-density approximation calculations show that the energy difference between pristine $\mathrm{ABA}$ and $\mathrm{ABC}$-stacking domains is $4 \mathrm{meV}$, which increases to $8 \mathrm{meV}$ after triazine decoration. When considering the van der Waals (vdW) correction by using the vdW density function, the energy difference is $35 \mathrm{meV}$ before decoration and $68 \mathrm{meV}$ after decoration. As a benchmark, the difference in total energies was compared between the ABAstacking and the ABC-stacking graphite by employing the adiabatic-connection fluctuation-dissipation theorem within random-phase approximation (ACFDT-RPA). The RPA results show that the total energy of the ABA-stacking form is $1 \mathrm{meV}$ per unit cell lower than that of the ABC-stacking form, consistent with standard DFT methods. Meanwhile, triazine molecules always adopt the C-top configuration, where the $\mathrm{N}$ atoms stay above the hollow of carbon rings in the top graphene layer. For the ABA domain, it means that the triazine molecules form a new fourth layer with $\mathrm{ABA}$-like stacking (that is, $\mathrm{ABAB}$ ). For the $\mathrm{ABC}$ domain, the fourth triazine layer combined with the underlying $\mathrm{ABC}$ trilayer graphene forms $\mathrm{ABCA}$ or $\mathrm{ABCB}$ configurations, which may be an intuitive explanation for the increased energy difference. These results suggest that the enlarged energy difference between $\mathrm{ABA}$ and $\mathrm{ABC}$ domains after triazine decoration provides a driving force for the transformation of $\mathrm{ABC}$ to $\mathrm{ABA}$ domains at low temperatures.

To explore the physical mechanism responsible for the observed $\mathrm{ABC}$ - to $\mathrm{ABA}$-domain transformation, this study conducted a simulation by using the density functional tight binding-molecular dynamics method. The possible stacking sequences in the $\mathrm{ABA} / \mathrm{ABC}$ sample are critical for the simulation and discussion. Figure 3 a schematically shows the three possible ways of constructing an $\mathrm{ABA} / \mathrm{ABC}$ sample. In the following discussion, to construct an $\mathrm{ABA} / \mathrm{ABC}$ domain structure, the carbon atoms between $\mathrm{ABA}$ and $\mathrm{ABC}$ domains must shift by $2.84+2.65 \times n \AA(n=0,1,2,3, \ldots)$ along the armchair direction, as shown in Fig. $3 \mathrm{~b}$, where $2.84 \AA$ equals two times the $\mathrm{C}-\mathrm{C}$ bond length. Model I represents a gapped $\mathrm{ABA} / \mathrm{ABC}$ trilayer, which consists of a continuous ABA-stacked $\mathrm{AB}$ bilayer with the additional $A$ layer and $C$ layer on top separated by a small gap. Model II is the grain boundary (GB) separated by the ABA/ABC trilayer, which consists of the $\mathrm{ABA}$ and $\mathrm{ABC}$ domains separated by a GB. GBs primarily consist of pentagon and heptagon pairs, and occasionally octagon rings to reconcile the connection of the two domains. Model III is the wrinkle-separated ABA/ABC trilayer, where the $\mathrm{ABA}$ and $\mathrm{ABC}$ domains are constructed from a continuous graphene layer. In this model, it is necessary to have a wrinkle separating the two domains to accommodate the $2.84+2.65 \times n \AA$ shift between the two stacking orders. Both Raman and AFM do not have a sufficiently high spatial resolution to differentiate between these models. However, simulations can 


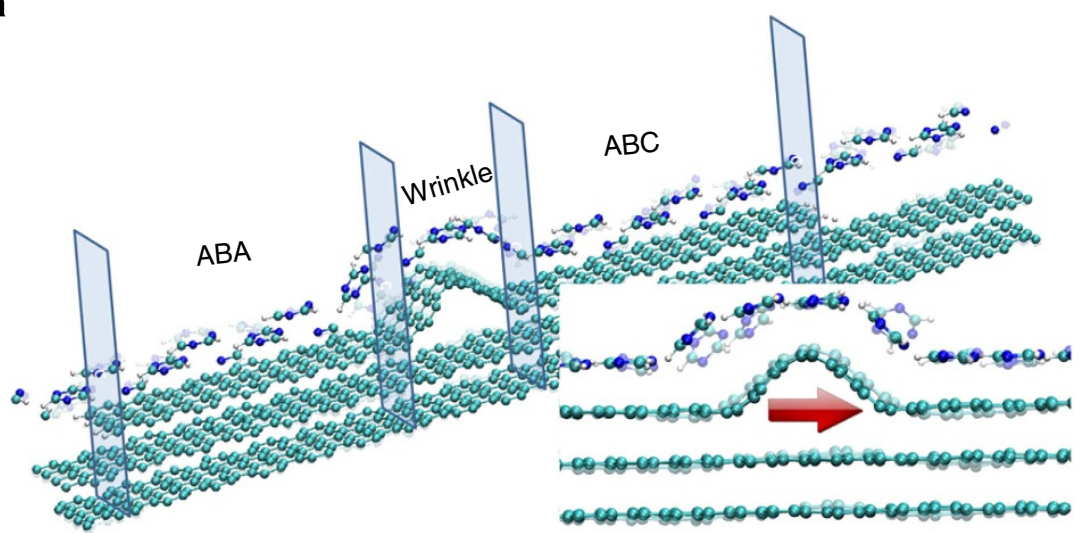

b

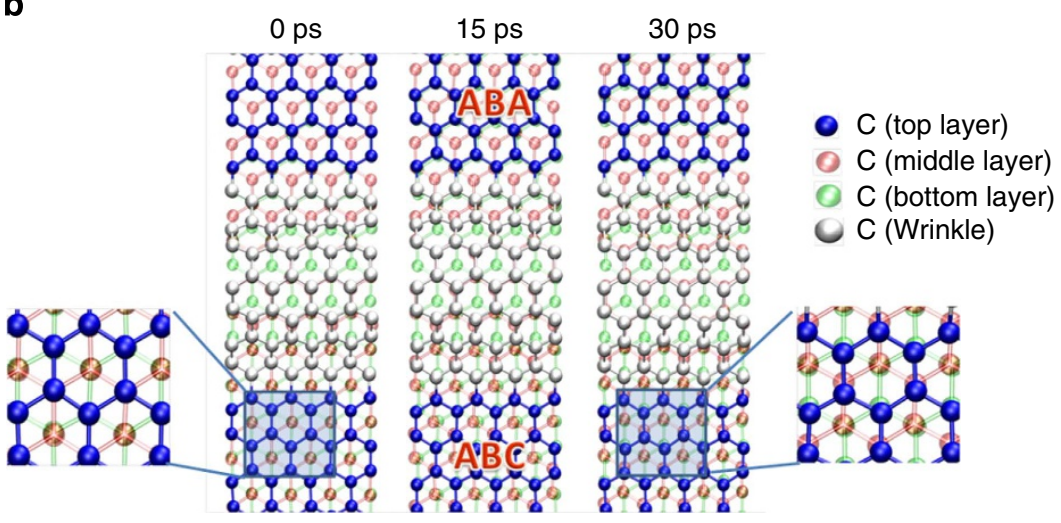

Figure 4 | Molecular dynamics simulations. The structural transformation of trilayer graphene decorated with triazine molecules at $500 \mathrm{~K}$. (a)

Structural evolution of the system between the initial model at 0 ps, (solid trace) and the structure at 30 ps (shaded trace). The simulated system contains an $A B A$ domain and an $A B C$ domain with a wrinkle, decorated with a single layer of triazine molecules. Inset: magnified plot around the wrinkle. The red arrow indicates that the wrinkle moves towards the $A B C$ domain direction, causing the instability of the $A B C$ domain. During the process, the triazine molecules on the top of the wrinkle show tilting, which act as a driving force to the wrinkle, leading to the wrinkle movement predominately towards the side of the ABC domain. Atomic colours are as follows: $\mathrm{H}$, white; N, blue; C, cyan. (b) Top-view snapshots of trilayer graphene (Top layer: blue; Middle layer: red; Bottom layer: green) near the wrinkle (white region). For clarity, the triazine molecules are not shown. The insets show the atomic configurations of the $\mathrm{ABC}$ domain at 0 and $30 \mathrm{ps}$. At $0 \mathrm{ps}$, the atoms in the bottom layer are all completely masked by those in the middle and top layers, while at $30 \mathrm{ps}$, the atoms in the top layer shift from their original position induced by the orientational change of the triazine molecules. For the ABA domain, the atomic arrangements do not show significant change.

help in understanding the transformation processes. We calculated the formation energies for the three possible configurations that contain both $\mathrm{ABA}$ and $\mathrm{ABC}$ domains, as shown in Fig. 3c. Formation energy is defined as $E_{\mathrm{f}}=E_{\text {system }}-m E_{\mathrm{C}}-n E_{\mathrm{H}}$, where $E_{\mathrm{C}}$ and $E_{\mathrm{H}}$ denote the energies of bulk graphite and the hydrogen molecule, respectively. As expected, the domains separated by point defects (Model I), such as the 5-5-8-5-5 GB, are energetically unfavourable compared with other models. This study adopted $n=0$ in calculating formation energy because the other case (that is, $n>0$ ) contains more defects and is less stable. Point defects (for example, vacancies and interstitials) can be trapped by the strained GB region, which in turn facilitates the migration of a GB in numerous samples, but has not been reported for graphene samples. Cong et al. ${ }^{24}$ reported that in one of their graphene trilayer samples, they observed tensile strain that induced $G$ and $2 D$ phonon softening at the $A B C$ and $A B A$ domain boundary ${ }^{31-34}$. However, the migration of $\mathrm{GB}$ in graphene is difficult because it requires the constant breaking and reforming of the $\mathrm{C}$ layer in the pentagon and heptagon rings. The diffusion and coalescence of vacancies in the GB region require a temperature of $2,000 \mathrm{~K}$, which is substantially higher than the temperature used in this study ${ }^{35}$. Hence, this model is highly unlikely, and can be excluded. For the wrinkle-separated
$\mathrm{ABA} / \mathrm{ABC}$ trilayer (Model III), a wrinkle was first made for the top-layer graphene, and the energy of the trilayer was minimized using a DFT calculation, showing that the separation using $n=3$ is the most stable structure. The gapped $\mathrm{ABA} / \mathrm{ABC}$ trilayer (Model I) is also made with $n=3$ for a fair comparison with Model I. The results show that Model III exhibits a similar formation energy compared with Model I.

The MD simulation in Fig. 4a shows that, when considering the triazine adsorption on the wrinkle-separated $\mathrm{ABA} / \mathrm{ABC}$ trilayer, the triazine molecules on top of the wrinkle region show tilting, which acts as a driving force to the wrinkle, leading the wrinkle to move toward the $\mathrm{ABC}$ domain direction, causing instability of the $\mathrm{ABC}$ domain. As shown in Fig. 4b, for the $\mathrm{ABA}$ domain, the atomic arrangements do not show a significant change. Compared with the stable $\mathrm{ABA}$ domain, the atomic lattice of the $\mathrm{C}$ layer in the $\mathrm{ABC}$ domain presents obvious slipping. The atoms in the top layer of the $\mathrm{ABC}$ domain shift from their original position, induced by the slight movement of the wrinkle, resulting in the final transformation of the $\mathrm{ABC}$ to ABA domains. However, for Model I, to explain the crystal growth-like behaviour for the $\mathrm{ABC}$ to $\mathrm{ABA}$ transformation observed in Fig. 2, the shift of the carbon atoms of the top-layer graphene from the edge of the $\mathrm{ABC}$ domain should result in an 
increased gap area, which is apparently not observed in our experiment. Thus, Model I is excluded. Combined with the experimental observation of a gradual $\mathrm{ABC}$ to $\mathrm{ABA}$ transformation, the structure with a wrinkle separating the $\mathrm{ABA}$ and $\mathrm{ABC}$ domains is the most plausible stacking sequence.

In summary, this work explores that the deposition of triazine molecules on top of the graphene trilayers results in the transformation of certain $\mathrm{ABC}$-stacked to ABA-stacked domains, which was confirmed by changes in the Raman $2 D, M$ and $G$ bands. This observation directly proves that the ABA-stacked graphene trilayer is thermodynamically more stable than the $\mathrm{ABC}$-stacked counterpart. The transformation of $\mathrm{ABC}$ to $\mathrm{ABA}$ domains started at the $\mathrm{ABA}-\mathrm{ABC}$ domain boundaries. The calculations show that after triazine decoration, the energy difference between the $\mathrm{ABA}$ and $\mathrm{ABC}$ domains is larger, providing a driving force for stacking transformation. The structure with a wrinkle separating the $\mathrm{ABA}$ and $\mathrm{ABC}$ domains is the most plausible $\mathrm{ABA}-\mathrm{ABC}$ domain boundary structure for the samples, and the triazine molecular decoration on the wrinkled graphene at the $\mathrm{ABC}-\mathrm{ABA}$ domain boundary activates the wrinkle movement toward the $\mathrm{ABC}$ domains, resulting in $\mathrm{ABA}$ domain growth and a stacking transformation of $\mathrm{ABC}$ to $\mathrm{ABA}$. The triazine-induced $\mathrm{ABC}$ - to $\mathrm{ABA}$-stacking transformation was also observed for thicker graphene layers such as tetralayers and hexa-layers (see Supplementary Fig. S4 for details). Although the details of the transformation from $\mathrm{ABC}$ - to the $\mathrm{ABA}$-stacking order for thicker graphene layers remain under investigation, the activation of top-layer wrinkles by triazine molecule decoration may also affect the structural property of graphene layers underneath, which could result in the rearrangement of the stacking order in few-layer graphene.

\section{Methods}

Sample preparation. Graphenes were mechanically exfoliated from natural graphite flakes (purchased from NGS) and then transferred to a substrate with $300 \mathrm{~nm}$ $\mathrm{SiO}_{2}$ on highly doped $\mathrm{Si}$. We verified the graphene layers using optical microscopy, Raman spectroscopy and AFM. From Raman 2D profiles, we did not observe turbostatic structure in our exfoliated trilayer samples. After characterization, the 1,3,5-triazine was thermally evaporated onto graphene films at $150^{\circ} \mathrm{C}$. The evaporation period was around $8 \mathrm{~h}$. The setup and process are the same to the reference ${ }^{30}$.

Characterization. The AFM images were performed in a Veeco Dimension-Icon system. Raman spectra were collected in a confocal Raman system (NT-MDT). The wavelength of laser is $473 \mathrm{~nm}(2.63 \mathrm{eV})$, and the spot size of the laser beam is $\sim 0.5 \mu \mathrm{m}$. The step size of Raman spatial mapping is $0.5 \mu \mathrm{m}$, and the spectral resolution is $3 \mathrm{~cm}^{-1}$ (obtained with a 600 -grooves per $\mathrm{mm}$ grating). A high grating $(1,800$ grooves per $\mathrm{mm})$ is also used to get more details of line shapes of Raman band, and the spectral resolution is $1 \mathrm{~cm}^{-1}$. The Si peak at $520 \mathrm{~cm}^{-1}$ was used as a reference for wavenumber calibration.

Simulation. For the simulation study, trilayer graphene flakes with a wrinkle GB were calculated by the density functional tight binding-molecular dynamics method ${ }^{36,37}$. The simulation system is coupled to an Andersen thermostat in order to maintain an invariant temperature of $500 \mathrm{~K}$. A small time step of $0.1 \mathrm{fs}$ is adopted to ensure the integration stability for the higher frequency $\mathrm{C}-\mathrm{H}$ stretch vibrations. The model containing 1,076 atoms was initially constructed according to the reliability. The triazine molecules are adopted with the most stable configurations for $\mathrm{ABC}$ and $\mathrm{ABA}$ domains, where carbon atoms in triazine molecules overlap with the graphene lattice. The vdW interaction between the atoms is taken into account via a Lennard-Jones potential. The first-principles DFT calculations to compute the total energies of systems are performed with the plane wave basis Vienna $a b$ initio simulation pack (VASP) code ${ }^{38}$. The electron-ion interaction was described using a projector augmented wave method and the exchange correlation potential was described using the local-density approximation in the parametrization of Perdew and Zunger ${ }^{39}$. We accounted for the non-local correlation energies by employing the vdW density functional method ${ }^{40}$. A kinetic energy cutoff at $400 \mathrm{eV}$ was adopted. As a benchmark, we calculated the energy difference between the ABA stacking and $\mathrm{ABC}$-stacking graphite employing the ACFDT-RPA using VASP ${ }^{41-43}$. Here, the in-plane lattice parameter and the interplane distance along the stacking direction is set to the experimental value of 2.46 and $3.34 \AA$. To ensure the convergence, we used high plane wave cutoffs of
$800 \mathrm{eV}$ both for the Hartree-Fock part and the correlation part ${ }^{44,45}$. For the HF part, $26 \times 26 \times 8$ k-grid was used. Results are shown in Supplementary Fig. S5.

\section{References}

1. Neto, A. H. C., Guinea, F., Peres, N. M. R., Novoselov, K. S. \& Geim, A. K. The electronic properties of graphene. Rev. Mod. Phys. 81, 109-162 (2009).

2. Ohta, T., Bostwick, A., Seyller, T., Horn, K. \& Rotenberg, E. Controlling the electronic structure of bilayer graphene. Science 313, 951-954 (2006).

3. Geim, A. K. \& Novoselov, K. S. The rise of graphene. Nat. Mater. 6, 183-191 (2007).

4. Zhang, W. J. \& Li, L. -J. Observation of phonon anomaly at the armchair edge of single-layer graphene in air. ACS Nano 5, 3347-3353 (2011).

5. Wu, Y. et al. High-frequency, scaled graphene transistors on diamond-like carbon. Nature 472, 74-78 (2011).

6. Kim, K. et al. Large-scale pattern growth of graphene films for stretchable transparent electrodes. Nature 457, 706-710 (2009).

7. Eda, G., Fanchini, G. \& Chhowalla, M. Large-area ultrathin films of reduced graphene oxide as a transparent and flexible electronic material. Nat. Nanotech 3, 270-274 (2008).

8. Schedin, F. et al. Detection of individual gas molecules adsorbed on graphene. Nat. Mater 6, 652-655 (2007).

9. Ang, P., Chen, W., Wee, A. T. S. \& Loh, K. P. Solution-gated epitaxial graphene as pH sensor. J. Am. Chem. Soc. 130, 14392-14393 (2008).

10. Mak, K. F., Shan, J. \& Heinz, T. F. Electronic structure of few-layer graphene: experimental demonstration of strong dependence on stacking sequence. Phys. Rev. Lett. 104, 176404-1-176404-4 (2010).

11. Craciun, M. F., Russo, S., Yamamoto, M., Oostinga, J. B., Morpurgo, A. F. \& Tarucha, S. Trilayer graphene is a semimetal with a gate-tunable band overlap. Nat. Nanotech 4, 383-388 (2009).

12. Zhang, F., Sahu, B., Min, H. \& MacDonald, A. H. Band structure of ABCstacked graphene trilayers. Phys. Rev. B 82, 035409-1-035409-10 (2010).

13. Lui, C. H., Li, Z., Mak, K. F., Cappelluti, E. \& Heinz, T. F. Observation of an electrically tunable band gap in trilayer graphene. Nat. Phys 7, 944-947 (2011).

14. Bao, W. et al. Stacking-dependent band gap and quantum transport in trilayer graphene. Nat. Phys 7, 948-952 (2011).

15. Zhang, L., Zhang, Y., Camacho, J., Khodas, M. \& Zaliznyak, I. The experimental observation of quantum Hall effect of $1=3$ chiral quasiparticles in trilayer graphene. Nat. Phys 7, 953-957 (2011).

16. Kumar, A. et al. Integer quantum hall effect in trilayer graphene. Phys. Rev. Lett 107, 126806-1-126806-4 (2011).

17. Lui, C. H., Li, Z. Q., Chen, Z. Y., Klimov, P. V., Brus, L. E. \& Heinz, T. F. Imaging stacking order in few-layer graphene. Nano Lett. 11, 164-169 (2011)

18. Grüneis, A. et al. Tight-binding description of the quasiparticle dispersion of graphite and few-layer graphene. Phys. Rev. B 78, 205425-1-205425-16 (2008)

19. Haering, R. R. Band structure of Rhombohedral graphite. Can. J. Phys. 36, 352-362 (1958)

20. Malard, L. M., Mafra, D. L., Doorn, S. K. \& Pimenta, M. A. Resonance Raman scattering in graphene: probing phonons and electrons. Solid State Commun. 149, 1136-1139 (2009).

21. Malard, L. M. et al. Electronic properties of bilayer graphene probed by resonance Raman scattering. Phys. Status Solid. B 245, 2060-2063 (2008)

22. Graf, D. et al. Spatially resolved Raman spectroscopy of single- and few-layer graphene. Nano Lett. 7, 238-242 (2007).

23. Ferrari, A. C. et al. Raman spectrum of graphene and graphene layers. Phys. Rev. Lett. 97, 187401-1-187401-4 (2006).

24. Cong, C., Yu, T., Saito, R., Dresselhaus, G. F. \& Dresselhaus, M. S. Second-order overtone and combination Raman modes of graphene layers in the range of 1690-2150 $\mathrm{cm}^{-1}$. ACS Nano 3, 1600-1605 (2011).

25. Rao, R. et al. Effects of layer stacking on the combination Raman modes in graphene. ACS Nano 5, 1594-1599 (2011).

26. Cong, C. et al. Raman characterization of ABA- and ABC-stacked trilayer graphene. ACS Nano 5, 8760-8768 (2011).

27. Cousins, C. S. G. Elasticity of carbon allotropes. IV. Rhombohedral graphite: Elasticity, zone-center optic modes, and phase transformation using transferred Keating parameters. Phys. Rev. B 67, 024110-1-024110-11 (2003).

28. Charlier, J. -C., Gonze, X. \& Michenaud, J. -P. First-principles study of the stacking effect on the electronic properties of graphite(s). Carbon. N. Y. 32, 289-299 (1994).

29. Aoki, M. \& Amawashi, H. Dependence of band structures on stacking and field in layered graphene. Sol. St. Com 142, 123-127 (2007).

30. Zhang, W. et al. Opening an electrical band gap of bilayer graphene with molecular doping. ACS Nano 5, 7517-7524 (2011).

31. Warner, J. H., Mukai, M. \& Kirland, A. I. Atomic structure of ABC Rhombohedral stacked trilayer graphene. ACS Nano 6, 5680-5686 (2012).

32. Huang, M. Y., Yan, H. G., Chen, C. Y., Song, D. H., Heinz, T. F. \& Hone, J. Phonon softening and crystallographic orientation of strained graphene studied by Raman spectroscopy. Proc. Natl Acad. Sci. USA 106, 7304-7308 (2009). 
33. Mohiuddin, T. M. G. et al. Uniaxial strain in graphene by Raman spectroscopy: G peak splitting, Grüneisen parameters and sample orientation. Phys. Rev. B 79, 205433-1-205433-8 (2009).

34. Ni, Z. H., Yu, T., Lu, Y. H., Wang, Y. Y., Feng, Y. P. \& Shen, Z. X. Uniaxial strain on graphene: Raman spectroscopy study and band-gap opening. ACS Nano 2, 2301-2305 (2008).

35. Wang, B., Puzyrev, Y. \& Pantelides, S. T. Strain enhanced defect reactivity at grain boundaries in polycrystalline graphene. Carbon 49, 3983-3988 (2011).

36. Aradi, B., Hourahine, B. \& Frauenheim, T. DFTB +, a sparse matrix-based implementation of the DFTB method. J. Phys. Chem. A 111, 5678-5684 (2007).

37. Elstner, M., Hobza, P., Frauenheim, T., Suhai, S. \& Kaxiras, E. Hydrogen bonding and stacking interactions of nucleic acid base pairs: a densityfunctional-theory based treatment. J. Chem. Phys. 114, 5149-5155 (2001).

38. Kresse, G. \& Furthmuller, J. Efficient iterative schemes for ab initio totalenergy calculations using a plane-wave basis set. Phys. Rev. B 54, 11169-11186 (1996).

39. Perdew, J. P. \& Zunger, A. Self-interaction correction to density-functional approximations for many-electron systems. Phys. Rev. B 23, 5048 (1981).

40. Klimeš, J., Bowler, D. R. \& Michaelides, A. Van der Waals density functionals applied to solids. Phys. Rev. B 81, 081408 (2010).

41. Vanin, M., Mortensen, J. J., Kelkkanen, A. K., Garcia-Lastra, J. M., Thygesen, K. S. \& Jacobsen, K. W. Graphene on metals: A van der Waals density functional study. Phys. Rev. B 81, 081408 (2010).

42. Marini, A., García-González, P. \& Rubio, A. First-principles description of correlation effects in layered materials. Phys. Rev. Lett. 96, 136404 (2006).

43. Harl, J. \& Kresse, G. Accurate bulk properties from approximate many-body techniques. Phys. Rev. Lett. 103, 056401 (2006).

44. Lebègue, S., Harl, J., Gould, T., Ángyán, J. G., Kresse, G. \& Dobson, J. F. Cohesive properties and asymptotics of the dispersion interaction in graphite by the random phase approximation. Phys. Rev. Lett. 105, 196401 (2010).

45. Olsen, T., Yan, J., Mortensen, J. J. \& Thygesen, K. S. Dispersive and covalent interactions between graphene and metal surfaces from the random phase approximation. Phys. Rev. Lett. 107, 156401 (2011).

\section{Acknowledgements}

This research was supported by Academia Sinica (IAMS and Nano program) and National Science Council Taiwan (NSC-99-2112-M-001-021-MY3 and 99-2738-M-001001). J.X.Y. and Z.X.S. acknowledge financial support from Singapore Ministry of Education Academic Research Fund Tier 3 under Grant number MOE2011-T3-1-005.

\section{Author contributions}

W.Z. performed the major part of the experiments. J.Y. performed simulation studies. C.S.C. helped to prepare graphene samples. All authors discussed the simulation results J.L.K. provided computation resources. L.J.L. and W.Z. conceived the study. L.J.L., W.Z. and J.Y. wrote the manuscript.

\section{Additional information}

Supplementary Information accompanies this paper at http://www.nature.com/ naturecommunications

Competing financial interests: The authors declare no competing financial interests.

Reprints and permission information is available online at http://npg.nature.com/ reprintsandpermissions/

How to cite this article: Zhang, W. et al. Molecular adsorption induces the transformation of rhombohedral- to Bernal-stacking order in trilayer graphene. Nat. Commun 4:2074 doi: $10.1038 /$ ncomms3074 (2013). 